\title{
A FETI Method for a Class of Indefinite or Complex Second- or Fourth-Order Problems
}

\author{
Charbel Farhat ${ }^{1}$, Jing $\mathrm{Li}^{2}$, Michel Lesoinne ${ }^{1}$ and Philippe Avery ${ }^{1}$ \\ 1 University of Colorado at Boulder, Department of Aerospace Engineering \\ Sciences (http://caswww. colorado.edu/ ${ }^{\sim}$ charbel/) \\ 2 Kent State University, Department of Mathematical Sciences
}

Summary. The FETI-DP domain decomposition method is extended to address the iterative solution of a class of indefinite problems of the form $\left(\mathbf{K}-\sigma^{\mathbf{2}} \mathbf{M}\right) \mathbf{x}=\mathbf{b}$, and a class of complex problems of the form $\left(\mathbf{K}-\sigma^{2} \mathbf{M}+i \sigma \mathbf{D}\right) \mathbf{x}=\mathbf{b}$, where $\mathbf{K}$, $\mathbf{M}$, and $\mathbf{D}$ are three real symmetric positive semi-definite matrices arising from the finite element discretization of either second-order elastodynamic problems or fourth-order plate and shell dynamic problems, $i$ is the imaginary complex number, and $\sigma$ is a positive real number.

\section{Introduction}

Real linear or linearized systems of equations of the form

$$
\left(\mathbf{K}-\sigma^{2} \mathbf{M}\right) \mathbf{x}=\mathbf{b}
$$

and complex linear or linearized systems of equations of the form

$$
\left(\mathbf{K}-\sigma^{2} \mathbf{M}+i \sigma \mathbf{D}\right) \mathbf{x}=\mathbf{b}
$$

are frequent in computational structural dynamics. Eq. (1) is encountered, for example, in the finite element (FE) simulation of the forced response of an undamped mechanical system to a periodic excitation. In that case, $\mathbf{K}$ and $\mathbf{M}$ are the FE stiffness and mass matrices of the considered mechanical system, respectively, $\sigma$ is the circular frequency of the external periodic excitation, $\mathbf{b}$ is its amplitude, $\left(\mathbf{K}-\sigma^{2} \mathbf{M}\right)$ is the impedance of the mechanical system, and $\mathbf{x}$ is the amplitude of its forced response. Such problems also arise during the solution by an inverse shifted method of the generalized symmetric eigenvalue problem $\mathbf{K} \mathbf{x}=\omega^{2} \mathbf{M x}$ associated with an undamped mechanical system. In that example, $\mathbf{K}$ and $\mathbf{M}$ have the same meaning as in the previous case, $\left(\omega^{2}\right.$, $\mathbf{x}$ ) is a desired pair of eigenvalue and eigenvector representing the square of a natural circular frequency and the corresponding natural vibration mode of the undamped mechanical system, respectively, and the shift $\sigma^{2}$ is introduced 
to obtain quickly the closest eigenvalues to $\sigma^{2}$. In both examples mentioned here, the matrices $\mathbf{K}$ and $\mathbf{M}$ are symmetric positive semi-definite, and therefore $\left(\mathbf{K}-\sigma^{\mathbf{2}} \mathbf{M}\right)$ rapidly becomes indefinite when $\sigma$ is increased. Eq. (2) is encountered in similar problems when the mechanical system is damped, in which case $i$ denotes the pure imaginary number satisfying $i^{2}=-1$ and $\mathbf{D}$ denotes the FE damping matrix and is also symmetric positive semi-definite.

Domain decomposition based preconditioned conjugate gradient (PCG) methods have emerged as powerful equation solvers in this field on both sequential and parallel computing platforms. While most successful domain decomposition methods (DDMs) have been designed for the solution of symmetric positive (semi-) definite systems, some have targeted indefinite problems of the form given in (1) (Cai and Widlund [1992]). The objective of this paper is to present an alternative DDM that addresses both classes of indefinite (1) and complex (2) problems, that is based on the FETI-DP (Farhat, Lesoinne and Pierson [2000], Farhat et al. [2001]) DDM, and that is scalable when $\mathbf{K}$, $\mathbf{M}$, and $\mathbf{D}$ result from the FE discretization of second-order elastodynamic problems and fourth-order plate and shell dynamic problems.

\section{The FETI-DP method}

The dual-primal finite element tearing and interconnecting method (FETIDP) (Farhat, Lesoinne and Pierson [2000], Farhat et al. [2001]) is a thirdgeneration FETI method (for example, see Farhat [1991], Farhat and Roux [1991]) developed for the scalable and fast iterative solution of systems of equations arising from the FE discretization of static, dynamic, second-order, and fourth-order elliptic partial differential equations (PDEs). When equipped with the Dirichlet preconditioner (Farhat, Mandel and Roux [1994]) and applied to fourth-order or two-dimensional second-order problems, the condition number $\kappa$ of its interface problem grows asymptotically as (Mandel and Tezaur [2001])

$$
\kappa=\mathcal{O}\left(1+\log ^{m} \frac{H}{h}\right), \quad m \leq 2,
$$

where $H$ and $h$ denote the subdomain and mesh sizes, respectively. When equipped with the same Dirichlet preconditioner and an auxiliary coarse problem constructed by enforcing some set of optional constraints at the subdomain interfaces (Farhat et al. [2001]), the condition number estimate (3) also holds for second-order scalar elliptic problems (Klawonn, Widlund and Dryja [2002]). The result (3) proves the numerical scalability of the FETI methodology with respect to all of the problem size, the subdomain size, and the number of subdomains. More specifically, it suggests that one can expect the FETI-DP method to solve small-scale and large-scale problems in similar iteration counts. This in turn suggests that when the FETI-DP method is well-implemented on a parallel processor, it should be capable of solving an $n$-times larger problem using an $n$-times larger number of processors in almost 
a constant CPU time. This was demonstrated in practice for many complex structural mechanics problems (for example, see Farhat, Lesoinne and Pierson [2000] and Farhat et al. [2001] and the references cited therein).

Next, the FETI-DP method is overviewed in the context of the generic symmetric positive semi-definite (static) problem

$$
\mathbf{K} \mathbf{x}=\mathbf{b},
$$

where $\mathbf{K}$ has the same meaning as in problems $(1,2)$ and $\mathbf{b}$ is an arbitrary vector, in order to keep this paper as self-contained as possible.

\subsection{Non-overlapping domain decomposition and notation}

Let $\Omega$ denote the computational support of a second- or fourth-order problem whose discretization leads to problem (4), $\left\{\Omega^{(s)}\right\}_{s=1}^{N_{s}}$ denote its decomposition into $N_{s}$ subdomains with matching interfaces $\Gamma^{(s, q)}=\partial \Omega^{(s)} \bigcap \partial \Omega^{(q)}$, and let $\Gamma=\bigcup_{s=1, q>s}^{s=N_{s}} \Gamma^{(s, q)}$ denote the global interface of this decomposition. In the remainder of this paper, each interface $\Gamma^{(s, q)}$ is referred to as an "edge", whether $\Omega$ is a two- or three-dimensional domain. Let also $\mathbf{K}^{(s)}$ and $\mathbf{b}^{(s)}$ denote the contributions of subdomain $\Omega^{(s)}$ to $\mathbf{K}$ and $\mathbf{b}$, respectively, and let $\mathbf{x}^{(s)}$ denote the vector of dof associated with it.

Let $N_{c}$ of the $N_{I}$ nodes lying on the global interface $\Gamma$ be labeled "corner" nodes (see Fig. 1), $\Gamma_{c}$ denote the set of these corner nodes, and let $\Gamma^{\prime}=\Gamma \backslash \Gamma_{c}$. If in each subdomain $\Omega^{(s)}$ the unknowns are partitioned into global corner dof designated by the subscript $c$, and "remaining" dof designated by the subscript $r, \mathbf{K}^{(s)}, \mathbf{x}^{(s)}$ and $\mathbf{b}^{(s)}$ can be partitioned as follows

$$
\mathbf{K}^{(s)}=\left[\begin{array}{cc}
\mathbf{K}_{r r}^{(s)} & \mathbf{K}_{r c}^{(s)} \\
\mathbf{K}_{r c}^{(s)^{T}} & \mathbf{K}_{c c}^{(s)}
\end{array}\right], \quad \mathbf{x}^{(s)}=\left[\begin{array}{c}
\mathbf{x}_{r}^{(s)} \\
\mathbf{x}_{c}^{(s)}
\end{array}\right] \quad \text { and } \quad \mathbf{b}^{(s)}=\left[\begin{array}{c}
\mathbf{b}_{r}^{(s)} \\
\mathbf{b}_{c}^{(s)}
\end{array}\right] .
$$

The $r$-type dof can be further partitioned into "interior" dof designated by the subscript $i$, and subdomain interface "boundary" dof designated by the subscript $b$. Hence, $\mathbf{x}_{r}^{(s)}$ and $\mathbf{b}_{r}^{(s)}$ can be further partitioned as follows

$$
\mathbf{x}_{r}^{(s)}=\left[\mathbf{x}_{i}^{(s)} \mathbf{x}_{b}^{(s)}\right]^{T} \quad \text { and } \quad \mathbf{b}_{r}^{(s)}=\left[\mathbf{b}_{i}^{(s)} \mathbf{b}_{b}^{(s)}\right]^{T},
$$

where the superscript $T$ designates the transpose.

Let $\mathbf{x}_{c}$ denote the global vector of corner dof, and $\mathbf{x}_{c}^{(s)}$ denote its restriction to $\Omega^{(s)}$. Let also $\mathbf{B}_{r}^{(s)}$ and $\mathbf{B}_{c}^{(s)}$ be the two subdomain Boolean matrices defined by

$$
\mathbf{B}_{r}^{(s)} \mathbf{x}_{r}^{(s)}= \pm \mathbf{x}_{b}^{(s)} \quad \text { and } \quad \mathbf{B}_{c}^{(s)} \mathbf{x}_{c}=\mathbf{x}_{c}^{(s)}
$$




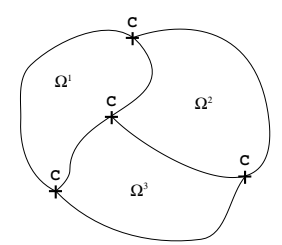

Fig. 1. Example of a definition of corner points.

where the \pm sign is set by any convention that implies that $\sum_{s=1}^{N_{s}} \mathbf{B}_{r}^{(s)} \mathbf{x}_{r}^{(s)}$ represents the jump of the solution $\mathbf{x}$ across the subdomain interfaces. Finally, let

$$
\mathbf{b}_{c}=\sum_{s=1}^{N_{s}} \mathbf{B}_{c}^{(s)^{T}} \mathbf{b}_{c}^{(s)} .
$$

In Farhat, Lesoinne and Pierson [2000] and Farhat et al. [2001], it was shown that solving problem (4) is equivalent to solving the following domaindecomposed problem

$$
\begin{aligned}
\mathbf{K}_{r r}^{(s)} \mathbf{x}_{r}^{(s)}+\mathbf{K}_{r c}^{(s)} \mathbf{B}_{c}^{(s)} \mathbf{x}_{c}+\mathbf{B}_{r}^{(s)^{T}} \lambda+\mathbf{B}_{r}^{(s)^{T}} \mathbf{Q}_{b} \mu & =\mathbf{b}_{r}^{(s)}, \quad s=1, \ldots, N_{s}(9) \\
\sum_{s=1}^{N_{s}} \mathbf{B}_{c}^{(s)^{T}} \mathbf{K}_{r c}^{(s)^{T}} \mathbf{x}_{r}^{(s)}+\sum_{s=1}^{N_{s}} \mathbf{B}_{c}^{(s)^{T}} \mathbf{K}_{c c}^{(s)} \mathbf{B}_{c}^{(s)} \mathbf{x}_{c} & =\mathbf{b}_{c}, \\
\sum_{s=1}^{N_{s}} \mathbf{B}_{r}^{(s)} \mathbf{x}_{r}^{(s)} & =0, \\
\mathbf{Q}_{b}^{T} \sum_{s=1}^{N_{s}} \mathbf{B}_{r}^{(s)} \mathbf{x}_{r}^{(s)} & =0,
\end{aligned}
$$

where $\lambda$ is an $N_{\lambda}$-long vector of Lagrange multipliers introduced on $\Gamma^{\prime}$ to enforce the continuity (11) of the solution $\mathbf{x}$, and $\mu$ is another vector of Lagrange multipliers introduced to enforce the optional linear constraints (12). These optional constraints, a concept first developed in Farhat, Chen, Risler and Roux [1998], are associated with a matrix $\mathbf{Q}_{b}$ with $N_{Q}<N_{\lambda}$ columns defined on $\Gamma^{\prime}$. The word "optional" refers to the fact that Eq. (12) and the vector of Lagrange multipliers $\mu$ are not necessarily needed for formulating the above domain-decomposed problem. Indeed, since the solution of problem (4) is continuous across the subdomain interfaces, it satisfies Eq. (11) and therefore satisfies Eq. (12) for any matrix $\mathbf{Q}_{b}$.

The domain-decomposed problem (9-12) was labeled "dual-primal" in Farhat, Lesoinne and Pierson [2000] and Farhat et al. [2001] because it is formulated in terms of two different types of global unknowns: the dual Lagrange multipliers represented by the vector $\lambda$, and the primal corner dof represented by the vector $\mathbf{x}_{c}$.

In the remainder of this paper, the $j$-th column of $\mathbf{Q}_{b}$ is denoted by $\mathbf{q}_{j}$. 


\subsection{Interface and coarse problems}

Let

$$
\begin{aligned}
\widetilde{\mathbf{K}}_{c c}=\left[\begin{array}{rr}
\mathbf{K}_{c c} & 0 \\
0 & 0
\end{array}\right], & \mathbf{d}_{r} & =\sum_{s=1}^{N_{s}} \mathbf{B}_{r}^{(s)} \mathbf{K}_{r r}^{(s)^{-1}} \mathbf{b}_{r}^{(s)}, \\
\text { and } & \mathbf{b}_{c}^{*} & =\mathbf{b}_{c}-\sum_{s=1}^{N_{s}}\left(\mathbf{K}_{r c}^{(s)} \mathbf{B}_{c}^{(s)}\right)^{T} \mathbf{K}_{r r}^{(s)-1} \mathbf{b}_{r}^{(s)}
\end{aligned}
$$

After some algebraic manipulations aimed at eliminating symbolically $\mathbf{x}_{r}^{(s)}$, $s=1, \ldots, N_{s}, \mathbf{x}_{c}$, and $\mu$, the domain-decomposed problem (9-12) can be transformed into the following symmetric positive semi-definite interface problem

$$
\left(\mathbf{F}_{I_{r r}}+\widetilde{\mathbf{F}}_{I_{r c}} \widetilde{\mathbf{K}}_{c c}^{*^{-1}} \widetilde{\mathbf{F}}_{I_{r c}}^{T}\right) \lambda=\mathbf{d}_{r}-\widetilde{\mathbf{F}}_{I_{r c}} \widetilde{\mathbf{K}}_{c c}^{*^{-1}} \tilde{\mathbf{b}}_{c}^{*},
$$

where

$$
\begin{aligned}
\mathbf{F}_{I_{r r}} & =\sum_{s=1}^{N_{s}} \mathbf{B}_{r}^{(s)} \mathbf{K}_{r r}^{(s)^{-1}} \mathbf{B}_{r}^{(s)^{T}}, \quad \widetilde{\mathbf{F}}_{I_{r c}}=\sum_{s=1}^{N_{s}} \mathbf{B}_{r}^{(s)} \mathbf{K}_{r r}^{(s)^{-1}} \widetilde{\mathbf{K}}_{r c}^{(s)} \mathbf{B}_{c}^{(s)}, \\
\widetilde{\mathbf{K}}_{r c}^{(s)} & =\left[\begin{array}{ll}
\mathbf{K}_{r c}^{(s)} \mathbf{B}_{c}^{(s)} & \mathbf{B}_{r}^{(s)^{T}} \mathbf{Q}_{b}
\end{array}\right], \quad \tilde{\mathbf{b}}_{c}^{*}=\left[\begin{array}{c}
\mathbf{b}_{c}^{*} \\
-\mathbf{Q}_{b}^{T} \mathbf{d}_{r}
\end{array}\right], \\
\widetilde{\mathbf{K}}_{c c}^{*} & =\widetilde{\mathbf{K}}_{c c}-\left[\begin{array}{c}
\sum_{s=1}^{N_{s}}\left(\mathbf{K}_{r c}^{(s)} \mathbf{B}_{c}^{(s)}\right)^{T} \mathbf{K}_{r r}^{(s)-1}\left(\mathbf{K}_{r c}^{(s)} \mathbf{B}_{c}^{(s)}\right) \sum_{s=1}^{N_{s}}\left(\mathbf{K}_{r c}^{(s)} \mathbf{B}_{c}^{(s)}\right)^{T} \mathbf{K}_{r r}^{(s)^{-1}}\left(\mathbf{B}_{r}^{(s)^{T}} \mathbf{Q}_{b}\right) \\
\sum_{s=1}^{N_{s}}\left(\mathbf{B}_{r}^{(s)^{T}} \mathbf{Q}_{b}\right)^{T} \mathbf{K}_{r r}^{(s)^{-1}}\left(\mathbf{K}_{r c}^{(s)} \mathbf{B}_{c}^{(s)}\right) \sum_{s=1}^{N_{s}}\left(\mathbf{B}_{r}^{(s)^{T}} \mathbf{Q}_{b}\right)^{T} \mathbf{K}_{r r}^{(s)^{-1}}\left(\mathbf{B}_{r}^{(s)^{T}} \mathbf{Q}_{b}\right)
\end{array}\right] .
\end{aligned}
$$

The FETI-DP method is a DDM which solves the original problem (4) by applying a PCG algorithm to the solution of the corresponding dual interface problem (14). At the $n$-th PCG iteration, the matrix-vector product $\left(\mathbf{F}_{I_{r r}}+\widetilde{\mathbf{F}}_{I_{r c}} \widetilde{\mathbf{K}}_{c c}^{*^{-1}} \widetilde{\mathbf{F}}_{I_{r c}}^{T}\right) \lambda^{n}$ incurs the solution of an auxiliary problem of the form

$$
\widetilde{\mathbf{K}}_{c c}^{*} \mathbf{z}=\widetilde{\mathbf{F}}_{I_{r c}}^{T} \lambda^{n}
$$

From the fifth of Eqs. (15), it follows that the size of this auxiliary problem is equal to the sum of the number of corner dof, $N_{c}^{\text {dof }}$, and the number of columns of the matrix $\mathbf{Q}_{b}, N_{Q}$.

For $N_{Q}=0$ - that is, for $\mathbf{Q}_{b}=0$, the auxiliary problem (16) is a coarse problem, and $\widetilde{\mathbf{K}}_{c c}^{*}$ is a sparse matrix whose pattern is that of the stiffness matrix obtained when each subdomain is treated as a "superelement" whose nodes are its corner nodes. This coarse problem ensures that the FETI-DP method equipped with the Dirichlet preconditioner (see Section 2.3) is numerically scalable for fourth-order plate and shell problems, and two-dimensional second-order elasticity problems (Farhat et al. [2001], Mandel and Tezaur 
[2001]). However, for $\mathbf{Q}_{b}=0$, the FETI-DP method equipped with the Dirichlet preconditioner is not numerically scalable for three-dimensional secondorder problems.

For any choice of $\mathbf{Q}_{b} \neq 0, \widetilde{\mathbf{K}}_{c c}^{*}$ remains a sparse matrix. If $\mathbf{Q}_{b}$ is constructed edge-wise - that is, if each column of $\mathbf{Q}_{b}$ is constructed as the restriction of some operator to a specific edge of $\Gamma^{\prime}$ - the sparsity pattern of $\widetilde{\mathbf{K}}_{c c}^{*}$ becomes that of a stiffness matrix obtained by treating each subdomain as a superelement whose nodes are its corner nodes augmented by virtual mid-side nodes. The number of dof attached to each virtual mid-side node is equal to the number of columns of $\mathbf{Q}_{b}$ associated with the edge on which lies this mid-side node. If $N_{Q}$ is kept relatively small, the auxiliary problem (16) remains a relatively small coarse problem. This coarse problem was labeled "augmented" coarse problem in Farhat, Lesoinne and Pierson [2000] in order to distinguish it from the smaller coarse problem obtained with $\mathbf{Q}_{b}=0$. Furthermore, each column of $\mathbf{Q}_{b}$ is referred to as an "augmentation coarse mode". When these augmentation coarse modes are chosen as the translational rigid body modes of each edge of $\Gamma^{\prime}$, the FETI-DP method equipped with the Dirichlet preconditioner becomes numerically scalable for three-dimensional second-order problems (Klawonn, Widlund and Dryja [2002]).

\subsection{Local preconditioning}

Two local preconditioners have been developed so far for the FETI-DP method:

1. The Dirichlet preconditioner which can be written as

$$
\begin{aligned}
\overline{\mathbf{F}}_{I_{r r}}^{D^{-1}} & =\sum_{s=1}^{N_{s}} \mathbf{W}^{(s)} \mathbf{B}_{r}^{(s)}\left[\begin{array}{ll}
0 & 0 \\
0 & \mathbf{S}_{b b}^{(s)}
\end{array}\right] \mathbf{B}_{r}^{(s)^{T}} \mathbf{W}^{(s)}, \\
\text { where } \quad \mathbf{S}_{b b}^{(s)} & =\mathbf{K}_{b b}^{(s)}-\mathbf{K}_{i b}^{(s)^{T}} \mathbf{K}_{i i}^{(s)^{-1}} \mathbf{K}_{i b}^{(s)},
\end{aligned}
$$

the subscripts $i$ and $b$ have the same meaning as in Section 2.1, and $\mathbf{W}^{(s)}$ is a subdomain diagonal scaling matrix that accounts for possible subdomain heterogeneities (Rixen and Farhat [1999]). This preconditioner is mathematically optimal in the sense that it leads to the condition number estimate (3).

2. The lumped preconditioner which can be written as

$$
\overline{\mathbf{F}}_{I_{r r}}^{L^{-1}}=\sum_{s=1}^{N_{s}} \mathbf{W}^{(s)} \mathbf{B}_{r}^{(s)}\left[\begin{array}{cc}
0 & 0 \\
0 & \mathbf{K}_{b b}^{(s)}
\end{array}\right] \mathbf{B}_{r}^{(s)^{T}} \mathbf{W}^{(s)} .
$$

This preconditioner is not mathematically optimal in the sense defined above; however, it decreases the cost of each iteration in comparison with the Dirichlet preconditioner often with a modest increase in the iteration count. 


\section{The FETI-DPH method}

In the context of Eq. (1), $\mathbf{K}_{r r}^{(s)}$ becomes $\mathbf{K}_{r r}^{(s)}-\sigma^{2} \mathbf{M}_{r r}^{(s)}$. Hence, the extension of the FETI-DP method to problems of the form given in (1) or (2) requires addressing the following issues:

1. $\mathbf{K}_{r r}^{(s)}-\sigma^{2} \mathbf{M}_{r r}^{(s)}$ is indefinite and therefore the dual interface problem (14) is indefinite.

2. Independently of which interface points are chosen as corner points, $\mathbf{K}_{r r}^{(s)}-$ $\sigma^{2} \mathbf{M}_{r r}^{(s)}$ is in theory singular when $\sigma^{2}$ coincides with an eigenvalue of the pencil $\left(\mathbf{K}_{r r}^{(s)}, \mathbf{M}_{r r}^{(s)}\right)$.

3. How to construct augmentation coarse modes and extended Dirichlet and lumped preconditioners that address the specifics of problems $(1,2)$.

For problems of the form given in (2), only the third issue is relevant. The first issue can be addressed by solving the dual interface problem (14) by a preconditioned generalized minimum residual (PGMRES) algorithm rather than a PCG algorithm. The second and third issues were addressed in Farhat, Macedo and Lesoinne [2000] in the context of the basic FETI method and acoustic scattering applications — that is, for the exterior Helmholtz scalar problem where $\sigma^{2}=k^{2}$ and $k$ denotes the wave number. More specifically, a regularization procedure was developed in that reference to prevent all subdomain problems from being singular for any value of the wave number $k$, without destroying the sparsity of the local matrices $\mathbf{K}_{r r}^{(s)}-k^{2} \mathbf{M}_{r r}^{(s)}$ and without affecting the solution of the original problem (1). Furthermore, for the scalar Helmholtz equation, the coarse modes were chosen in Farhat, Macedo and Lesoinne [2000] as plane waves of the form $e^{i k \theta_{j}^{T} X_{b}}, j=1,2, \cdots$, where $\theta_{j}$ denotes a direction of wave propagation and $X_{b}$ the coordinates of a node on $\Gamma$. The resulting DDM was named the FETI-H method ( $\mathrm{H}$ for Helmholtz).

Unfortunately, the regularization procedure characterizing the FETI-H method transforms each real subdomain problem associated with Eq. (1) into a complex subdomain problem. For acoustic scattering applications, this is not an issue because the Sommerfeld radiation condition causes the original problem to be in the complex domain. However, for real-valued problems such as those represented by Eq. (1), the regularization procedure of the FETI-H method is unjustifiable from computational resource and performance viewpoints.

In practice, experience reveals that $\mathbf{K}_{r r}^{(s)}-\sigma^{2} \mathbf{M}_{r r}^{(s)}$ is non-singular as long as $\mathbf{K}_{r r}^{(s)}$ is non-singular. This observation is exploited here to design an extension of the FETI-DP method for indefinite problems of the form given in (1) and complex problems of the form given in (2) by:

1. Replacing the PCG solver by the PGMRES solver.

2. Adapting the Dirichlet and lumped preconditioners to exploit an interesting characteristic of problems $(1,2)$. 
3. Constructing a new augmentation coarse space that is effective for secondorder elastodynamic problems as well as fourth-order plate and shell dynamic problems.

The extension of FETI-DP outlined above is named here the FETI-DPH method.

\subsection{Adapted Dirichlet and lumped preconditioners}

Consider the subdomain (impedance) matrix

$$
\mathbf{Z}_{r r}^{(s)}=\mathbf{K}_{r r}^{(s)}-\sigma^{2} \mathbf{M}_{r r}^{(s)}
$$

For the applications outlined in the introduction, $\mathbf{M}_{r r}^{(s)}$ is a mass matrix; hence, in three dimensions and at the element level, this matrix is proportional to $h^{3}$. On the other hand, for the same applications, $\mathbf{K}_{r r}^{(s)}$ is a stiffness matrix; in three dimensions and at the element level, it is proportional to $h$ for second-order elasticity problems, and to $1 / h^{2}$ for fourth-order plate and shell problems. It follows that for a sufficiently fine mesh, $\mathbf{Z}_{r r}^{(s)}$ is dominated by $\mathbf{K}_{r r}^{(s)}$. These observations, the optimality of the Dirichlet preconditioner and the computational efficiency of the lumped preconditioner established for the solution of problem (4) suggest preconditioning the local matrices $\mathbf{Z}_{r r}^{(s)}$ by Dirichlet and lumped constructs that are based on $\mathbf{K}_{r r}^{(s)}$ (see Section 2.3) and not $\mathbf{Z}_{r r}^{(s)}$. When Rayleigh damping is used,

$$
\mathbf{D}_{r r}^{(s)}=c_{K} \mathbf{K}_{r r}^{(s)}+c_{M} \mathbf{M}_{r r}^{(s)}
$$

where $c_{K}$ and $c_{M}$ are two real constants, and the same reasoning can be invoked to advocate preconditioning the local matrices

$$
\mathbf{Z}_{r r}^{(s)}=\mathbf{K}_{r r}^{(s)}-\sigma^{2} \mathbf{M}_{r r}^{(s)}+i \sigma \mathbf{D}_{r r}^{(s)}
$$

by Dirichlet and lumped constructs that are based on $\left(1+i \sigma c_{K}\right) \mathbf{K}_{r r}^{(s)}$ and not $\mathbf{Z}_{r r}^{(s)}$.

Finally, it is pointed out that the ad-hoc reasoning outlined above can be mathematically justified, at least in the context of the scalar Helmholtz equation (for example, see Klawonn [1995] and the references cited therein).

\subsection{Wavy augmented coarse problem}

Let $\mathbf{r}$ denote the residual associated with the iterative solution of the dual interface problem (14). From Eqs. (9-12) and Eq. (14), it follows that

$$
\mathbf{r}=\mathbf{d}_{r}-\widetilde{\mathbf{F}}_{I_{r c}} \widetilde{\mathbf{K}}_{c c}^{*^{-1}} \tilde{\mathbf{b}}_{c}^{*}-\left(\mathbf{F}_{I_{r r}}+\widetilde{\mathbf{F}}_{I_{r c}} \widetilde{\mathbf{K}}_{c c}^{*^{-1}} \widetilde{\mathbf{F}}_{I_{r c}}^{T}\right) \lambda=\sum_{s=1}^{N_{s}} \mathbf{B}_{r}^{(s)} \mathbf{x}_{r}^{(s)}
$$


which shows that the residual $\mathbf{r}$ represents the jump of the iterate solution across the subdomain interfaces.

From Eq. (12), Eq. (15), Eq. (14) and Eq. (11), it follows that at each iteration of the PGMRES algorithm applied to the solution of problem (14), FETI-DPH forces the jump of the solution across the subdomain interfaces to be orthogonal to the subspace represented by the matrix $\mathbf{Q}_{b}$. This feature is a strategy for designing an auxiliary coarse problem which, when $\mathbf{Q}_{b}$ is well chosen, accelerates the convergence of a DDM (Farhat, Chen, Risler and Roux [1998]). In this work, the search for a suitable matrix $\mathbf{Q}_{b}$ is driven by the following reasoning. Suppose that the space of traces on $\Gamma^{\prime}$ of the desired solution of problem (1) is spanned by a set of orthogonal vectors $\left\{\mathbf{v}_{j_{E}}\right\}_{j=1}^{N_{\lambda}}$, where the subscript $E$ indicates that $\mathbf{v}_{j_{E}}$ is non-zero only on edge $E \in \Gamma^{\prime}$. Then, the residual $\mathbf{r}$ defined in Eq. (22) can be written as

$$
\mathbf{r}=\sum_{j=1}^{N_{\lambda}} \alpha_{j} \mathbf{v}_{j_{E}}
$$

where $\left\{\alpha_{j}\right\}_{j=1}^{N_{\lambda}}$ is a set of real coefficients. If each augmentation coarse mode is chosen as

$$
\mathbf{q}_{j}=\mathbf{v}_{j_{E}}, \quad j=1, \cdots, N_{Q}
$$

Eq. (12) simplifies to

$$
\alpha_{j}=0, \quad j=1, \cdots, N_{Q}
$$

In that case, Eq. (25) implies that at each iteration of the PGMRES algorithm, the first $N_{Q}$ components of the residual $\mathbf{r}$ in the basis $\left\{\mathbf{v}_{j_{E}}\right\}_{j=1}^{N_{\lambda}}$ are zero. If a few vectors $\left\{\mathbf{v}_{j_{E}}\right\}_{j=1}^{N_{Q}}, N_{Q}<<N_{\lambda}$, that dominate the expansion (23) can be found, then choosing these vectors as coarse augmentation modes can be expected to accelerate the convergence of the iterative solution of the dual interface problem (14). Hence, it remains to exhibit such a set of orthogonal vectors $\mathbf{v}_{j_{E}}$ and construct a computationally efficient matrix $\mathbf{Q}_{b}$.

A second-order elastodynamic problem is governed by Navier's displacement equations of motion

$$
\mu \Delta u+(\Lambda+\mu) \nabla(\nabla \cdot u)+b=\rho \frac{\partial^{2} u}{\partial t^{2}},
$$

where $u \in \mathbb{R}^{3}$ denotes the displacement (vector) field of the elastodynamic system, $\Lambda$ and $\mu$ its Lamé moduli, $b \in \mathbb{R}^{3}$ its body forces, $\rho$ its density, and $t$ denotes time. If a harmonic motion is assumed, - that is, if

$$
u(X, t)=v(X) e^{-i \omega t},
$$

where $X \in \mathbb{R}^{3}$ denotes the spatial variables, and $\omega$ denotes a circular frequency, the homogeneous form of Eq. (26) becomes 


$$
\mu \Delta v+(\Lambda+\mu) \nabla(\nabla \cdot v)+\rho \omega^{2} v=0 .
$$

The free-space solutions of the above vector equation are

$$
\begin{array}{ll}
v=a_{p} \sin \left(k_{p} \theta \cdot X\right), & v=a_{p} \cos \left(k_{p} \theta \cdot X\right), \\
v=a_{s_{1}} \sin \left(k_{s} \theta \cdot X\right), & v=a_{s_{1}} \cos \left(k_{s} \theta \cdot X\right), \\
v=a_{s_{2}} \sin \left(k_{s} \theta \cdot X\right), & v=a_{s_{2}} \cos \left(k_{s} \theta \cdot X\right),
\end{array}
$$

where $\theta \in \mathbb{R}^{3}$ is an arbitrary vector of unit length $\left(\|\theta\|_{2}=1\right), a_{p} \in \mathbb{R}^{3}$ is a vector that is parallel to $\theta,\left(a_{s_{1}}, a_{s_{2}}\right) \in \mathbb{R}^{3} \times \mathbb{R}^{3}$ are two independent vectors in the plane orthogonal to $\theta$,

$$
k_{p}=\sqrt{\frac{\rho \omega^{2}}{\Lambda+2 \mu}}, \quad \text { and } \quad k_{s}=\sqrt{\frac{\rho \omega^{2}}{\mu}} .
$$

The free-space solutions (29) are known as the elastic pressure or longitudinal waves, and the free-space solutions (30) and (31) are known as the elastic shear or transverse waves.

Consider next the following fourth-order PDE associated with a given elastic body

$$
\Delta^{2} u-\frac{m}{D} \omega^{2} u=0, \quad \text { where } \quad m=\rho \tau, \quad D=\frac{E \tau^{3}}{12\left(1-\nu^{2}\right)},
$$

$E$ denotes the Young modulus of the elastic body, $\nu$ its Poisson ratio, $\tau$ its thickness, and all other variables have the same meaning as before. The reader can check that the free-space solutions $(29,30,31)$ with

$$
k_{p}=k_{s}=\sqrt[4]{\frac{m}{D} \omega^{2}}
$$

are also free-space solutions of Eq. (33). The PDE (33) can model the harmonic transverse motion of a plate. In that case, $u$ is a scalar representing the transverse displacement field. However, for the purpose of constructing an augmented coarse problem for the FETI-DPH method, and only for this purpose, it is assumed here that when $u \in \mathbb{R}^{3}$, Eq. (33) models the harmonic motion of a shell in all three dimensions.

Hence, a general solution of either Eq. (28) or Eq. (33) can be written as

$$
\begin{aligned}
v & =\sum_{j=1}^{\infty}\left\{a_{p_{j}}\left(c_{1_{j}} \sin \left(k_{p} \theta_{j} \cdot X\right)+c_{2_{j}} \cos \left(k_{p} \theta_{j} \cdot X\right)\right)\right\} \\
& +\sum_{j=1}^{\infty}\left\{a_{s_{1_{j}}}\left(c_{3_{j}} \sin \left(k_{s} \theta_{j} \cdot X\right)+c_{4_{j}} \cos \left(k_{s} \theta_{j} \cdot X\right)\right)\right\} \\
& +\sum_{j=1}^{\infty}\left\{a_{s_{2 j}}\left(c_{5_{j}} \sin \left(k_{s} \theta_{j} \cdot X\right)+c_{6_{j}} \cos \left(k_{s} \theta_{j} \cdot X\right)\right)\right\}
\end{aligned}
$$


where $\theta_{j} \in \mathbb{R}^{3}$ is an arbitrary vector of unit length defining the direction of propagation of an elastic pressure or shear wave, $c_{1_{j}}, c_{2_{j}}, c_{3_{j}}, c_{4_{j}}, c_{5_{j}}$, and $c_{6_{j}}$ are real coefficients, and $k_{p}$ and $k_{s}$ are given by Eq. (32) for a secondorder elastodynamic problem and by Eq. (34) for a fourth-order plate or shell dynamic problem. From Eq. (35) and Eq. (24), it follows that the desired matrix $\mathbf{Q}_{b}$ is composed of blocks of six columns. The columns of each block are associated with one direction of propagation $\theta_{j}$ and one edge $E$ of the mesh partition, and can be written as

$$
\begin{aligned}
\mathbf{q}_{b_{l}}\left[\begin{array}{c}
3(m-1)+1 \\
3(m-1)+2 \\
3(m-1)+3
\end{array}\right] & =a_{p_{j}} \sin \left(k_{p} \theta_{j} \cdot X_{m}\right), \quad \mathbf{q}_{b_{l+1}}\left[\begin{array}{c}
3(m-1)+1 \\
3(m-1)+2 \\
3(m-1)+3
\end{array}\right]=a_{p_{j}} \cos \left(k_{p} \theta_{j} \cdot X_{m}\right), \\
\cdots & \\
\mathbf{q}_{b_{l+4}}\left[\begin{array}{c}
3(m-1)+1 \\
3(m-1)+2 \\
3(m-1)+3
\end{array}\right] & =a_{s_{2_{j}}} \sin \left(k_{s} \theta_{j} \cdot X_{m}\right), \quad \mathbf{q}_{b_{l+5}}\left[\begin{array}{l}
3(m-1)+1 \\
3(m-1)+2 \\
3(m-1)+3
\end{array}\right]=a_{s_{2_{j}}} \cos \left(k_{s} \theta_{j} \cdot X_{m}\right), \\
l & =6(j-1)+1, \quad m=1, \cdots, N_{I}-N_{c},
\end{aligned}
$$

where $\mathbf{q}_{b}[3(m-1)+1]$ designates the entry of $\mathbf{q}_{b}$ associated with the dof in the $x$-direction attached to the $m$-th node on an edge $E \in \Gamma^{\prime}, \mathbf{q}_{b}[3(m-$ $1)+2]$ designates the entry associated with the dof along the $y$-direction, $\mathbf{q}_{b}[3(m-1)+3]$ designates the entry associated with the dof along the $z$ direction, and $X_{m} \in \mathbb{R}^{3}$ denotes the coordinates of this $m$-th node. Hence, if $N_{E}$ denotes the number of edges of the mesh partition, and $N_{\theta}$ the number of considered directions of wave propagation, the total number of augmentation coarse modes is given in general by $N_{Q}=6 N_{E} N_{\theta}$. To these modes can be added the edge-based translational rigid body modes as these are free-space solutions of Eq. (28) when $\omega=0$.

In this paper, the number of directions is limited by $N_{\theta}^{\max }=13$, and the directions $\theta_{j}$ are generated as follows. A generic cube is discretized into $3 \times 3 \times 3$ points. A direction $\theta_{j}$ is defined by connecting the center point to any of the other 26 points lying on a face of the cube. Since each direction $\theta_{j}$ is used to define both a cosine and a sine mode, only one direction $\theta_{j}$ is retained for each pair of opposite directions, which results in a maximum of 13 directions.

\section{Performance studies and preliminary conclusions}

Here, the FETI-DPH method is applied to the solution of various problems of the form given in (1) or (2) and associated with: (a) the discretization by quadratic tetrahedral elements (10 nodes per element) of a wheel carrier fixed at a few of its nodes, and (b) the discretization by linear triangular shell elements of an alloy wheel clamped at a few center points (Fig. 2). When the structure is assumed to be damped, Eq. (20) is used to construct $D$ and $c_{K}$ 
and $c_{M}$ are determined by requiring that the critical damping ratio of the first 10 modes of the structure be equal in a least-squares sense to a specified value, $\xi$. In all problems, the shift is set to $\sigma^{2}=\omega^{2}=4 \pi^{2} f^{2}$, where $\omega^{2}$ is the square of a (possibly natural) circular frequency of the structure and $f$ is the corresponding frequency in Hz. To help the reader appreciate the magnitude of a chosen shift value, the natural frequencies of both structures are characterized in Table 1 . In order to investigate the performance, potential, and various scalability properties of the FETI-DPH method, various values of $\sigma^{2}$ are considered, three meshes with different resolutions are employed for the wheel carrier second-order problem (504,375 dof, 1,317,123 dof, and 2,091,495 dof), and one mesh with 936,102 dof is employed for the alloy wheel fourthorder shell problem. In all cases, the right-sides of problems $(1,2)$ are generated by a distributed load, the computations are performed on a Silicon Graphics Origin 3800 system with $40 \mathrm{R} 12000400 \mathrm{MHz}$ processors, and convergence is declared when the relative residual satisfies

$$
R E^{n}=\frac{\left\|\left(\mathbf{K}-\sigma^{2} \mathbf{M}+i \sigma \mathbf{D}\right) \mathbf{x}^{n}-\mathbf{b}\right\|_{2}}{\|\mathbf{b}\|_{\mathbf{2}}} \leq 10^{-6} .
$$
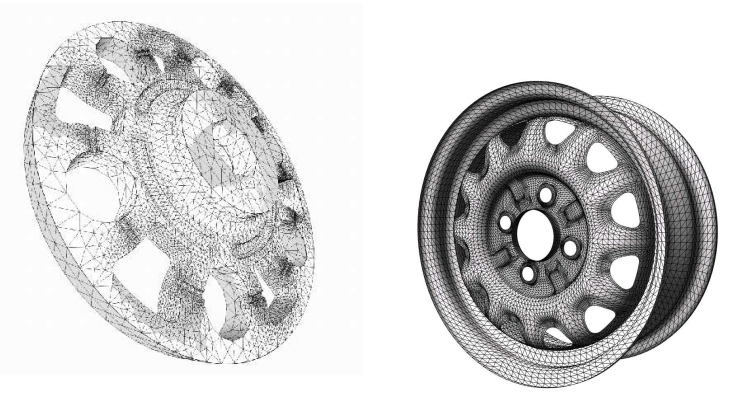

Fig. 2. FE discretizations of a wheel carrier (left) and an alloy wheel (right).

First, attention is directed to the wheel carrier undamped problem, and for each generated mesh, $N_{s}$ is chosen to keep the subdomain problem size constant. Two frequencies, $500 \mathrm{KHz}$ and $2 \mathrm{MHz}$, are considered: the latter value of the shift $\sigma^{2}$ arises, for example, when exciting the structure by its $200-t h$ natural frequency, or shifting around it during the solution of an eigenvalue problem. The number of wave directions is set to $N_{\theta}=2$, and the three translational rigid body modes are included in the construction of the augmentation matrix $\mathbf{Q}_{b}$. The performance results of the FETI-DPH solver obtained on $N_{p}=12$ processors are reported in Table 2 where $N_{i t r}$ records the iteration count. For each considered frequency, the iteration count associated with the chosen number of subdomains and chosen preconditioner is almost independent of the mesh size, which highlights the numerical scalability of the FETI-DPH method with respect to both the subdomain problem 
Table 1. Eigenvalue/Frequency partial spectrum of the pencil $(\mathbf{K}, \mathbf{M})$.

\begin{tabular}{||c|c|c||c|c||}
\hline \hline & Wheel Carrier & $\left(2^{n d}\right.$-order $)$ & Alloy Wheel & $\left(4^{t h}\right.$-order $)$ \\
\hline Mode Number & Eigenvalue $\left(\omega^{2}\right)$ & Frequency & Eigenvalue $\left(\omega^{2}\right)$ & Frequency \\
\hline \hline 1 & $2.6 \mathrm{e}+11$ & $8.2 \mathrm{e}+04 \mathrm{~Hz}$ & $7.6 \mathrm{e}+05$ & $1.4 \mathrm{e}+02 \mathrm{~Hz}$ \\
200 & $5.2 \mathrm{e}+13$ & $1.1 \mathrm{e}+06 \mathrm{~Hz}$ & $1.0 \mathrm{e}+09$ & $5.1 \mathrm{e}+03 \mathrm{~Hz}$ \\
300 & $1.6 \mathrm{e}+14$ & $2.0 \mathrm{e}+06 \mathrm{~Hz}$ & $3.0 \mathrm{e}+09$ & $8.7 \mathrm{e}+03 \mathrm{~Hz}$ \\
400 & $2.8 \mathrm{e}+14$ & $2.6 \mathrm{e}+06 \mathrm{~Hz}$ & $5.7 \mathrm{e}+09$ & $1.2 \mathrm{e}+04 \mathrm{~Hz}$ \\
500 & $4.0 \mathrm{e}+14$ & $3.2 \mathrm{e}+06 \mathrm{~Hz}$ & $9.5 \mathrm{e}+09$ & $1.5 \mathrm{e}+04 \mathrm{~Hz}$ \\
600 & $5.1 \mathrm{e}+14$ & $3.5 \mathrm{e}+06 \mathrm{~Hz}$ & & \\
\hline \hline
\end{tabular}

size and the total problem size. For this second-order problem, the lumped and Dirichlet preconditioners deliver similar CPU performances; hence, the lumped preconditioner is preferable since it requires less memory.

Table 2. Performance of the FETI-DPH solver: wheel carrier, undamped, $2^{\text {nd }}$-order problem; fixed subdomain problem size; $N_{\theta}=2$ ( + the three translational rigid body modes); $N_{p}=12$.

\begin{tabular}{||l|r|r|r|r|r|r|r||}
\hline \hline Frequency & Shift $\left(\sigma^{2}\right)$ & Mesh size & $N_{s}$ & $\begin{array}{r}N_{i t r} \\
\text { Lumped }\end{array}$ & $\begin{array}{r}\text { CPU } \\
\text { Lumped }\end{array}$ & $\begin{array}{r}N_{i t r} \\
\text { Dirichlet }\end{array}$ & $\begin{array}{r}\text { CPU } \\
\text { Dirichlet }\end{array}$ \\
\hline \hline \multirow{2}{*}{$5 \times 10^{5} \mathrm{~Hz}$} & \multirow{2}{*}{$9.8 \mathrm{e}+12$} & 504,375 dof & 250 & 63 & $64 \mathrm{~s}$. & 45 & $60 \mathrm{~s}$. \\
& & $1,317,123$ dof & 600 & 70 & $207 \mathrm{~s}$. & 53 & $206 \mathrm{~s}$. \\
& $2,091,495$ dof & 950 & 60 & $364 \mathrm{~s}$. & 45 & $358 \mathrm{~s}$. \\
\hline \multirow{3}{*}{$2 \times 10^{6} \mathrm{~Hz}$} & \multirow{2}{*}{$1.6 \mathrm{e}+14$} & 504,375 dof & 250 & 137 & $123 \mathrm{~s}$. & 105 & $119 \mathrm{~s}$ \\
& & $1,317,123$ dof & 600 & 174 & $483 \mathrm{~s}$. & 140 & $491 \mathrm{~s}$. \\
& $2,091,495$ dof & 950 & 151 & $901 \mathrm{~s}$. & 118 & $887 \mathrm{~s}$. \\
\hline \hline
\end{tabular}

To illustrate the performance of the FETI-DPH solver for problems of the form given in (2), the wheel carrier is next assumed to have a Rayleigh damping. The mesh with $N_{\text {dof }}=1,317,123$ is considered, the number of subdomains is set to $N_{s}=600$, the shift is set to $\sigma^{2}=10^{5} \mathrm{~Hz}$, the number of wave directions is set to $N_{\theta}=2$, the three translational rigid body modes are included in the construction of the augmentation matrix $\mathbf{Q}_{b}$, and the number of processors is set to $N_{p}=16$. For these parameters, the performance results of FETI-DPH equipped with the lumped preconditioner are reported in Table 3 for the undamped case $(\xi=0)$, and for realistic damping scenarios $(\xi=1 \%, \xi=2 \%$, and $\xi=5 \%)$. These results suggest that the intrinsic performance of FETI-DPH improves with the amount of damping. For the undamped case, FETI-DPH operates in the real domain. This explains why in that case, each iteration is 2.7 times faster than in the damped case where FETI-DPH operates in the complex plane. 
Table 3. Performance of the FETI-DPH solver: wheel carrier, damped, $2^{\text {nd }}$-order problem; $N_{\text {dof }}=1,317,123 ; N_{s}=600 ; \sigma^{2}=10^{5} \mathrm{~Hz}$; lumped preconditioner; $N_{\theta}=2$ ( + the three translational rigid body modes); $N_{p}=16$.

\begin{tabular}{||r|r|r|r|r||}
\hline \hline$\xi$ & $c_{K}$ & $c_{M}$ & $N_{i t r}$ & CPU \\
\hline \hline $0 \%$ & 0 & 0 & 62 & $182 \mathrm{~s} . \mid$ \\
\hline \hline $1 \%$ & $3.42 \mathrm{e}-6$ & 17.9 & 51 & $403 \mathrm{~s}$. \\
\hline $2 \%$ & $6.85 \mathrm{e}-6$ & 35.8 & 49 & $394 \mathrm{~s} . \mid$ \\
\hline $5 \%$ & $1.71 \mathrm{e}-5$ & 89.5 & 48 & $384 \mathrm{~s}$. \\
\hline \hline
\end{tabular}

Next, attention is directed to the undamped alloy wheel problem to investigate the performance for a fourth-order shell problem of the FETI-DPH solver equipped with the Dirichlet preconditioner. Two different frequencies, $5 \mathrm{KHz}$ and $20 \mathrm{KHz}$, are considered: the upper value of the shift $\sigma^{2}$ arises, for example, when exciting the considered alloy wheel by a frequency that is higher than its 400-th natural frequency, or shifting around that frequency during the solution of an eigenvalue problem. The number of subdomains is varied between $N_{s}=100$ and $N_{s}=400$ and the number of processors is fixed to $N_{p}=8$. Table 4 , where $N_{\text {coarse }}$ denotes the total size of the augmented coarse problem, contrasts for each value of $N_{s}$ the performance of FETI-DP (with PGMRES as a solver) and the best performance of FETI-DPH obtained by varying $N_{\theta}$. The reported performance results suggest that the FETI-DPH solver is numerically scalable for dynamic shell problems of the form given in (1). They also highlight the superiority of FETI-DPH over FETI-DP which fails to converge in a reasonable iteration count for large values of the shift $\sigma^{2}$.

Table 4. FETI-DPH vs. FETI-DP: alloy wheel, undamped, $4^{\text {th }}$-order problem; $N_{\text {dof }}=936,102 ;$ Dirichlet preconditioner; $N_{p}=8$.

\begin{tabular}{|c|c|c|c|c|c|c|}
\hline Frequency & Shift $\left(\sigma^{2}\right)$ & $N_{s}$ & $N_{\theta}$ & $N_{\text {coarse }}$ & $N_{i t r}$ & $\mathrm{CPU}$ \\
\hline \multirow{6}{*}{$5 \times 10^{3} \mathrm{~Hz}$} & \multirow{6}{*}{$9.8 \mathrm{e}+8$} & 100 & 0 & 3,258 & 347 & $534 \mathrm{~s}$. \\
\hline & & 100 & 3 & 7,275 & 122 & $265 \mathrm{~s}$. \\
\hline & & 200 & 0 & 6,372 & 236 & $301 \mathrm{~s}$. \\
\hline & & 200 & 2 & 11,853 & 116 & $200 \mathrm{~s}$. \\
\hline & & 400 & 0 & 12,129 & 226 & $317 \mathrm{~s}$. \\
\hline & & 400 & 2 & 21,924 & 123 & $271 \mathrm{s.}$ \\
\hline \multirow{6}{*}{$2 \times 10^{4} \mathrm{~Hz}$} & \multirow{6}{*}{$1.6 \mathrm{e}+10$} & 100 & 0 & 3,258 & $>400$ & \\
\hline & & 100 & 5 & 9,512 & 330 & $680 \mathrm{~s}$. \\
\hline & & 200 & 0 & 6,372 & $>400$ & \\
\hline & & 200 & 5 & 17,581 & 261 & $564 \mathrm{s.}$ \\
\hline & & 400 & 0 & 12,129 & $>400$ & \\
\hline & & 400 & 3 & 27,270 & 265 & $706 \mathrm{~s}$. \\
\hline
\end{tabular}


Acknowledgement. This research was supported by the Sandia National Laboratories under Contract No. 31095. The first author also acknowledges the support by the Sandia National Laboratories under Contract No. 29341, and the second author also acknowledges partial support by the National Science Foundation under Grant No. DMS-0209297. Any opinions, findings, and conclusions or recommendations expressed in this material are those of the authors and do not necessarily reflect the views of the Sandia National Laboratories or the National Science Foundation.

\section{References}

X. C. Cai and O. Widlund. Domain decomposition algorithms for indefinite elliptic problems. SIAM J. Sci. Statist. Comput., 13:243-258, 1992.

C. Farhat, M. Lesoinne, and K. Pierson. A scalable dual-primal domain decomposition method. Numer. Lin. Alg. Appl., 7:687-714, 2000.

C. Farhat, M. Lesoinne, P. LeTallec, K. Pierson, and D. Rixen. FETI-DP: a dual-primal unified FETI method - part I: a faster alternative to the two-level FETI method. Internat. J. Numer. Meths. Engrg., 50:1523-1544, 2001.

C. Farhat. A Lagrange multiplier based divide and conquer finite element algorithm. J. Comput. Sys. Engrg., 2:149-156, 1991.

C. Farhat and F. X. Roux. A method of finite element tearing and interconnecting and its parallel solution algorithm, Internat. J. Numer. Meths. Engrg., 32:1205-1227, 1991.

C. Farhat, J. Mandel and F. X. Roux. Optimal convergence properties of the FETI domain decomposition method. Comput. Meths. Appl. Mech. Engrg., 115:367-388, 1994.

J. Mandel and R. Tezaur, On the convergence of a dual-primal substructuring method. Numer. Math., 88:543-558, 2001.

A. Klawonn, O. B. Widlund and M. Dryja. Dual-primal FETI methods for three-dimensional elliptic problems with heterogeneous coefficients. SIAM J. Numer. Anal., 40:159-179, 2002.

C. Farhat, P. S. Chen, F. Risler and F. X. Roux. A unified framework for accelerating the convergence of iterative substructuring methods with Lagrange multipliers. Internat. J. Numer. Meths. Engrg., 42:257-288, 1998.

D. Rixen and C. Farhat. A simple and efficient extension of a class of substructure based preconditioners to heterogeneous structural mechanics problems. Internat. J. Numer. Meths. Engrg., 44:489-516, 1999.

C. Farhat, A. Macedo and M. Lesoinne. A two-level domain decomposition method for the iterative solution of high frequency exterior Helmholtz problems. Numer.Math., 85:283-308, 2000.

A. Klawonn. Preconditioners for Indefinite Problems. Ph. D. Thesis, Westfalische, Wilhelms-Universitat, Munster, 1995. 